\title{
Load Balancing in Cloud Computing with Enhanced Genetic Algorithm
}

\author{
Kalpana, Manjula Shanbhog
}

\begin{abstract}
Cloud computing has a decentralized architecture in which virtual machine migration is one of the major challenges which affects the network performance. To balance the network load, various techniques are designed for the virtual machine migration. In the previous research work, genetic algorithm was proposed for Virtual Machine (VM) migration which can balance the network load. The genetic algorithm is complex in nature which increases the execution time. In this research work, genetic algorithm is improved for VM migration which reduces the execution time and also space and bandwidth utilization.
\end{abstract}

KEYWORDS: Genetic Algorithm, Virtual Machine migration, Bandwidth utilization.

\section{INTRODUCTION}

Cloud Computing is service which provides on-demand and simple access of the network to several servers which provides computing resources like applications, storage, networks are in presence for other services which cloud provides which we can use to gain maximum efficiency. User retrieved data and modified data which is stored by client or an organization in centralized data called cloud. Cloud is a design, where cloud service provider provides services to user on demand and this vital feature is known as CSP stands for "Cloud Service Provider". Cloud computing gives Shared pool of assets (computers resources like networks, server and storage) on the demand of the user in ubiquitous and in simple word provides usability to the end customer maintaining minimum cost for their usage. According to NIST definition the basic concept of cloud computing can be easily understood [1]. From the above definition, we can conclude that cloud computing provides virtual infrastructure backed by software and hardware resources available on the internet. Cloud computing gives access to a user's shared pool of resources on demand of the user, to which the user subscribe and use for the time he wants to use and this all is achieved with the help of virtualization, which further helps in reducing the cost of implementing or adding more hardware parts to achieve the requirements of the user [2]. In cloud computing, there is no need to know the physical location, configuration of the system which provides the service. The basic features of cloud computing are: virtualization, homogeneity, modern security, on demand scale, minimum cost software, geographic distribution service orientation. One can use the application without installation and just by accessing internet user can manage

Revised Manuscript Received on July 22, 2019.

Kalpana, IILM Academy of Higher Learning College of Engineering \& Technology, Greater Noida, India.

Manjula Shanbhog, IILM Academy of Higher Learning College of Engineering \& Technology, Greater Noida, India. their personal files at any location through cloud computing. Using feature of centralizing storage cloud computing provides more efficient computing and resource allocation, bandwidth and memory [3]. Cloud provides elastic, scalable and shared resources to user which helps to provide resources to the client. In recent times cloud computing is an enhance era which affords distinct services like software as a resource, hardware as a resource, database as a resource, network as a resource, monitoring as a resource, infrastructure as a resource and having your complete platform as a resource, more over human as a resource. Deployment model of Cloud computing has been categorized into four categories. Three categories are mainly used today. Public cloud is known as a most common model of cloud computing to all clients that is an open cloud to model. In this model, cloud services are provided through virtualized environment developed using polled shared physical resources and share on public network such as internet. Same infrastructures shared by multiple clients [4]. Through this model, operations under cloud are performed optimally. Private Cloud is designed and developed under the needs of single organization. Private cloud service provider gives you access to its network in a more secure way insuring that anyone outside of your network won't be able to access it. This makes the private cloud more secure but less flexible considering public cloud. Hybrid cloud is collection of many clouds like private cloud and public cloud. All clouds have their own unique identities, but they all are determinate as a unit. It offers standardized get to information and application [5]. While working with private clouds sometimes we need some more storage space so we use some of public clouds this is known as cloud bursting. In this case organization will only have to pay for the extra space being consumed. Load balancing is a technique where the complete load of the network is shared using various techniques amongst the different nodes to make resource utilization efficient and to enhance the response time of the job. In the meantime, remove a state in which some of the hubs are under stacked while a few others are over stacked. Using many components while trying to balance load instead of a single component the reliability and availability of the data can be increased through redundancy [6]. This load is measured in terms of statistics around CPU load, memory used, delay or network load. A program or an operating system on a virtual space within a host acting as an individual entity in itself catering to request and providing services like a physical system is called a Virtual Machine, despite being virtual within a host it acts as a complete system by itself. A virtual machine is usually created inside a bigger environment referred as host. A host can have multiple virtual machines acting as an independent

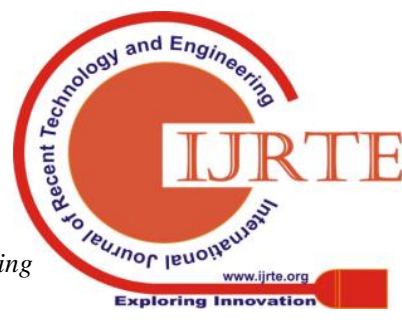


entity in itself. Process of switching a request from one Virtual machine to another without end user getting aware of it is called Virtual Machine Migration [7].

It can be done for several reasons, most importantly done for load balancing, disaster recovery, systematic upgrades and uninterrupted service. Genetic Algorithm began from the investigations of cell automata. It is led by John Holland and his partners. Fundamentally Genetic Algorithm is one of the seeking methods it is utilized as a part of the computer science. It finds solution for any optimization issues. The genetic algorithms are known as the evolutionary algorithms.

\section{Literature REVIEW}

Sheetal Karki et al. in 2018 [8] explains that the data is stored in a centralized virtual machine called cloud and the cloud provider companies are responsible to assign the offerings to the end users. The end users get entry to the offerings primarily based on their needs and are to be paid for what's being served. As the number of requests grows so the need for load balancing arises to maximize the useful resource utilization and energy consumption. Threshold and Check Pointing algorithm help in task migration when the virtual machines get overloaded at the time of cloudlet execution. The tasks are migrated from one virtual machine to another or can be queued to be decided by threshold and check pointing algorithm minimizing the processing time, energy and resource consumption.

Sukhpreet Kaur et al. in 2017 [9] proposed an Improved Genetic Algorithm (IGA) for assigning the users task to the virtual machines. The agenda of this proposed method is to maximize resource utilization while maintaining minimized energy consumption and reduce the task execution cost. All of the responsibilities are assigned to the available VMs in a manner such that the weight is balanced by distributing the dynamic workload across a couple of virtual machines to make certain that no single VM is either over applied or underutilized. The graphical representation of the simulation shows that the Improved Genetic Algorithm is far more efficient than the existing Genetic Algorithm in terms of several parameters such as energy efficiency and cost and also depicts that all the VMs are allocated to the cloudlets in Improved Genetic algorithm while in Genetic Algorithm some VMs are not allocated to any of the Cloudlet.

WANG Bei et al. in 2016 [10] stated a Multi-Population Genetic Algorithm (MPGA) thinking about load balancing is followed for solving the task scheduling issues in cloud environment in place of Genetic Algorithm to keep away the earlier convergence. In order to enlarge the search efficiency, the min-min and max-min algorithm are used for the populace initialization. Moreover, Metropolis criterion is used here to display the offspring so that poor individuals can also be accepted with a certain chance, then the population diversity can be maintained and the local optimum can also be avoided. The simulation outcomes show that a higher task scheduling result can be carried out through the MPGA-based task scheduling algorithm, which means the algorithm can realize an effective task scheduling and is more appropriate for managing portions of tasks in comparison to adaptive genetic algorithm.
Mahalingam et al. in 2015 [11] stated that one of the essential element for efficient operation in cloud computing is balancing the load. In this paper, the weight based optimized load balancing technique is proposed for the distribution of incoming jobs uniformly over various virtual machines or servers. The performance is analyzed by using the cloud simulator and comparing the current result with existing Round Robin and EIRP algorithms. Load has been uniformly distributed under the simulation results demonstrated by proposed algorithm. It may also implement a enhance service broker policy in the simulator to enhance new algorithm in the simulator.

Keke Gai et al. in 2015 [12] defines several earlier researches that have explored the optimizations of on-premise heterogeneous memories. Although, the heterogeneous cloud memories are having constraints because of the price and overall performance obstacles as a result of the hardware distributions and manipulative mechanisms. This paper works in this problem and proposes a singular technique, cost-aware Heterogeneous Cloud Memory Model (CAHCM), aiming to offer an excessive-overall performance cloud-based heterogeneous memory carrier presenting. The main algorithm helping CAHCM is Dynamic data Allocation advance (2DA) algorithm that makes use of genetic programming to conclude allocations of data at the cloud-based memories. Ultimately, we enforce experimental reviews to study our proposed model. Outcomes of the research have explored that this technique is feasible for being a cost-aware cloud-based solution.

Mayur S. Pilavare et al. in 2015 [13] states that as cloud computing is connected via network with servers so there are many issues to be solved. Load balancing is the crucial issue over the cloud to be addressed. The Genetic Algorithm outperforms some existing load balancing techniques. By giving the prioritized input to the genetic algorithm the response time will be decreased and this minimizes the make span of given task set. Here the jobs are assumed having same priority that may not be the actual case so it can be taken for further work and the various selection techniques for GA can be changed for better performance and crossover and mutation techniques can be modified to get better performance.

\section{RESEARCH Methodology}

All the free virtual machines available within the system are identified by improved genetic algorithm. The availability of free virtual machine is checked whenever a new task arrives. The task is allocated to the particular virtual machine in case when a virtual machine is available. However, in case no virtual machine is available, the machine whose task will be completed at the earliest is assigned the next task. Therefore, a proper utilization of all the VMs is done and an idle or over utilization condition is avoided here. Thus, with respect to cost and energy efficiency, the results achieved here are better as compared to other existing techniques.

Following are the various steps of Improved Genetic Algorithm: 
1. Initialization: The initial populace of candidate solutions is normally generated arbitrarily across the search area. However, the domain-specific knowledge or other information can be easily incorporated.

2. Evaluation: As soon as the population is initialized or an offspring populace is created, the evaluation of the fitness values of the candidate solutions takes place.

3. Selection: Those proposed with better fitness values are given more copies of the selection and for this reason the survival-of-the-fittest mechanism is imposed on the candidate solutions. Better selection is chosen over the worse one is the idea here and people have tried multiple strategies of selection theory to accomplish this idea, including stochastic universal selection there are others like roulette-wheel selection and ranking selection and few uncommon selections like tournament selection, these are well described in the following report.

4. Recombination: Recombination merge the components of two or more parental solutions to create new, possibly better solutions (i.e. offspring). There are many approaches of carrying out this and competent overall performance depends on a well designed recombination mechanism. The offspring beneath recombination will not be same to any particular parent and will alternatively integrate parental developments in a novel manner.

The process of implementation can be applied in following steps:

Step1: In the first step, the cloud network is deployed with the finite number of virtual machines.

Step 2: In the second step, the best virtual machine is selected for the cloudlet execution and when the fault occurs in the network the next step is executed.

Step 3: The improved genetic algorithm is executed which reassign the task to some other virtual machine when the fault occurs and further execution takes place.

\section{Pseudo Code of Improved Genetic Algorithm}

Begin

Step 1: Get list of all VMs working on all hosts.

Step 2: Initialize no migration is done.

Step 3: Get failure rate, resource utilization, and execution time of all machines.

Step 4: Built transition matrix for hosts and VMs.

Step 5: Loop will execute until all machines on over utilized hosts are migrated.

Step 5.1: For the specific VM that requires migration, the current usage of each host is to be calculated

Step 5.2: The creation history of VM is to be checked.

Step 5.3: Compare increase in utilization of selected hosts with other hosts.

Step 5.4: The host for which increase in usage is minimum is selected and then loop is ended.

Step 5.6: Repeat step 5.1 in case the maximum usage exceeds upper usage chromosome value.

Step 6: Else, the specific host for migration is selected.

Step 7: return migration List

END

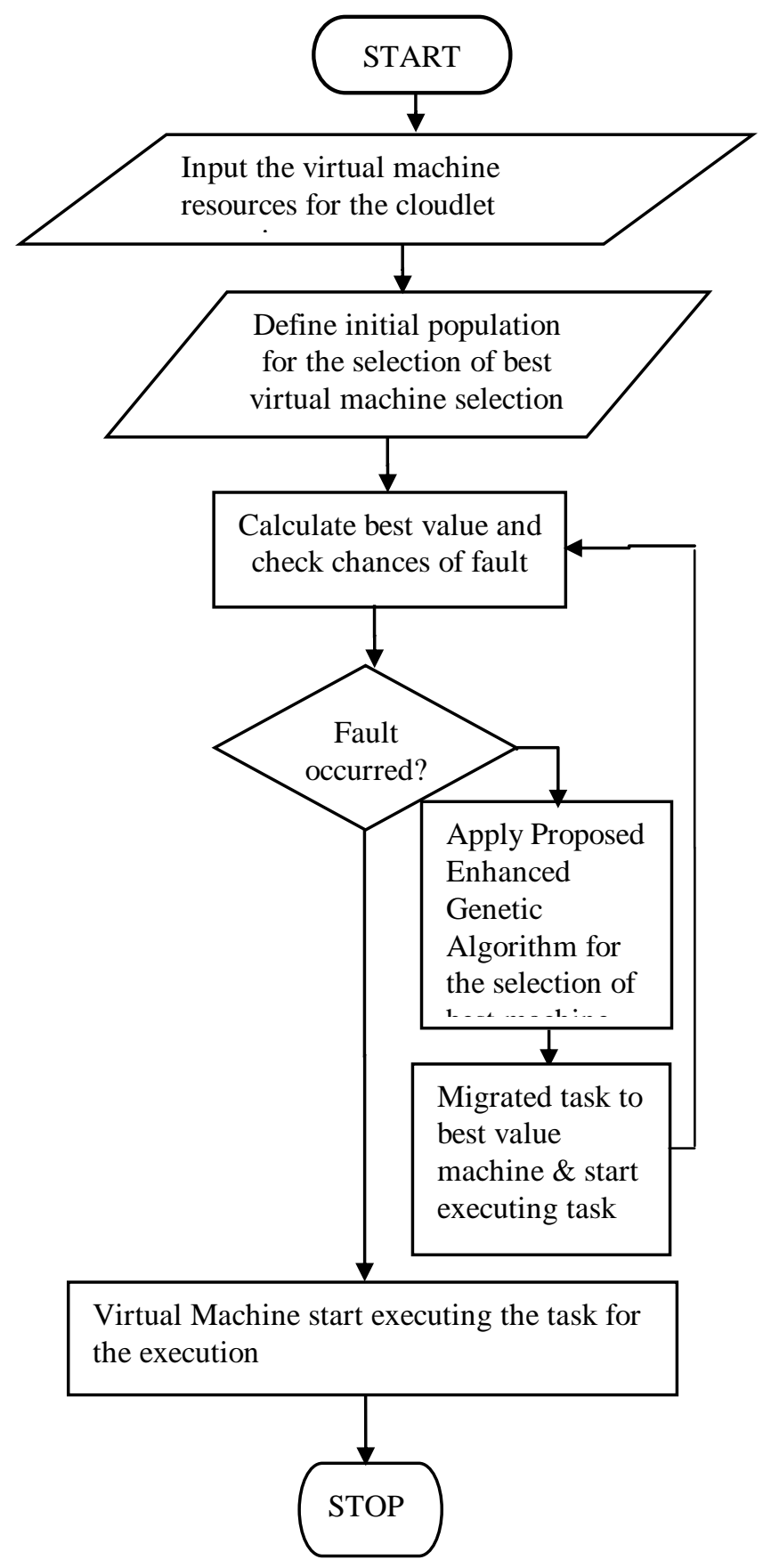

Fig 1: FLOWCHART OF IMPROVED GENETIC ALgORITHM

\section{EXPERIMENTAL RESULTS}

The proposed approach is implemented in MATLAB and the results are evaluated by comparing the proposed and existing techniques in terms of various parameters. 
Load Balancing in Cloud Computing with Enhanced Genetic Algorithm

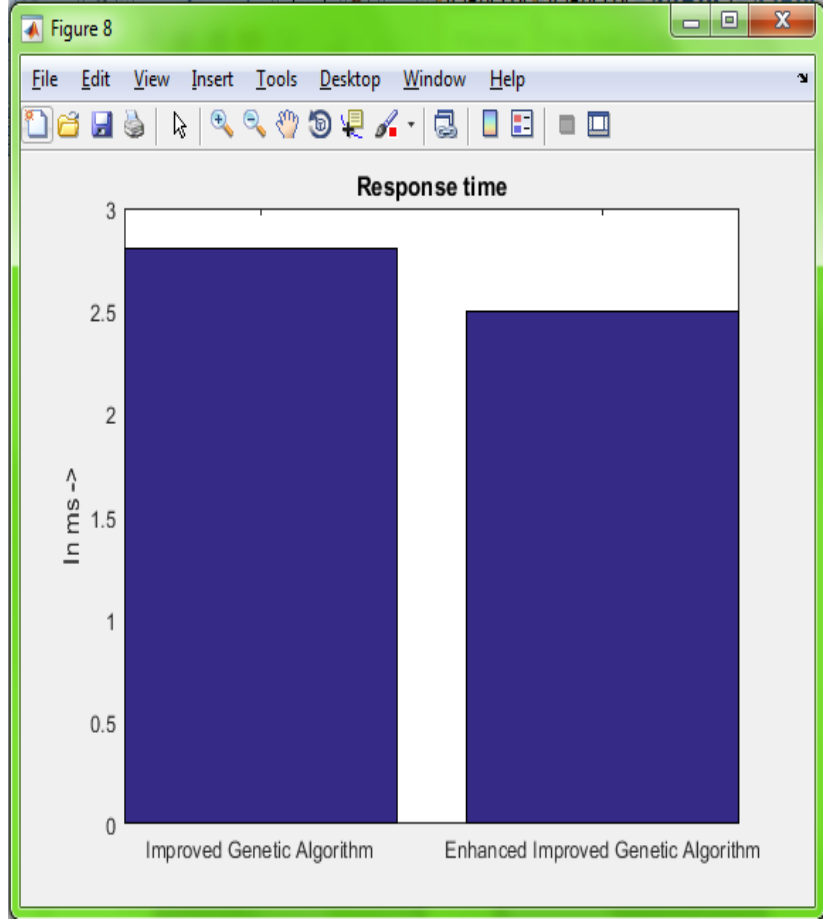

Fig 2: Comparison graph of Response Time

Figure 2 shows the response time of the improved genetic algorithm and proposed enhanced improved genetic algorithm compared for the performance analysis. The response time of enhanced improved genetic algorithm is less as compared to improved genetic algorithm.

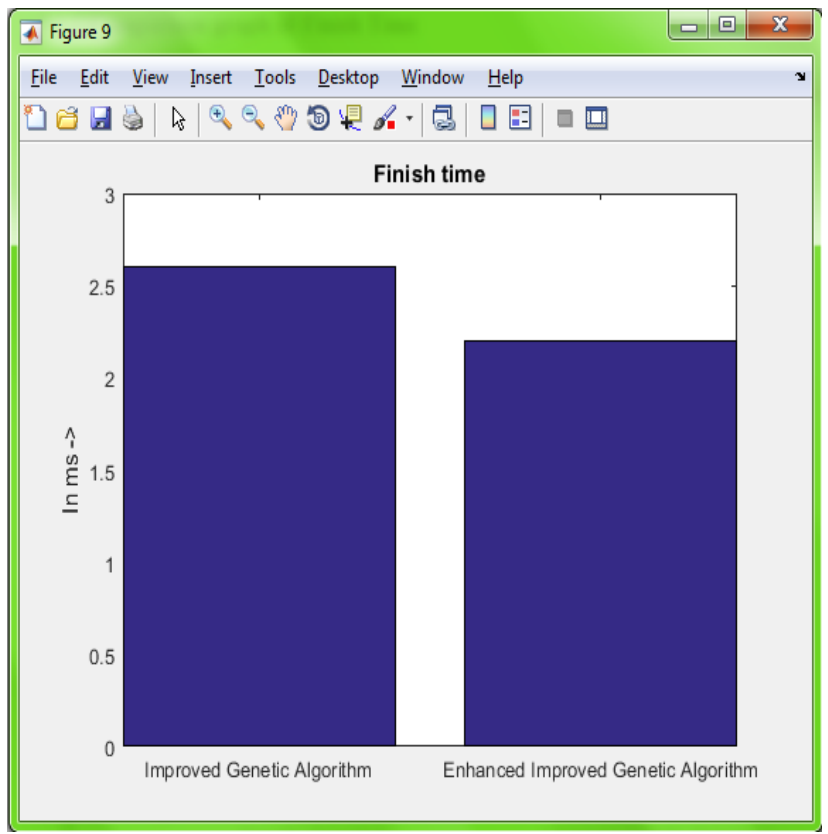

Fig 3: Comparison graph of Finish Time

Figure 3 shows the finish time of the improved genetic and proposed enhanced improved genetic algorithm compared for the performance analysis. The finish time of the enhanced improved genetic algorithm is less as compared to improved genetic Algorithm.

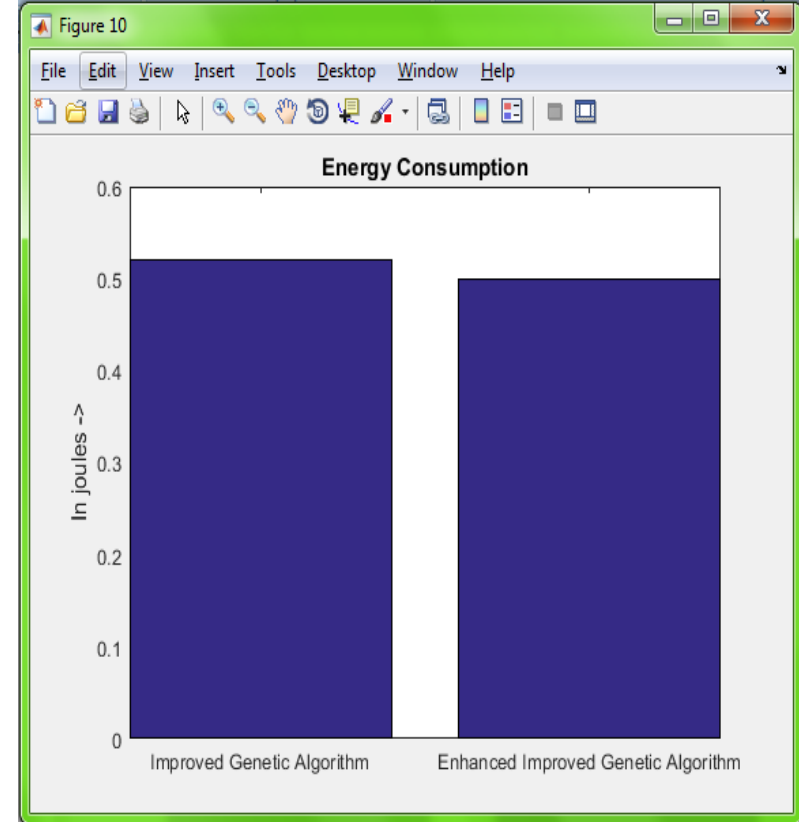

Fig 4: Comparison graph of Energy Consumption

Figure 4 shows the energy consumption of the improved genetic algorithm and proposed enhanced improved genetic algorithm compared for the performance analysis. The energy consumption of enhanced improved genetic algorithm is less as compared to improved genetic algorithm.

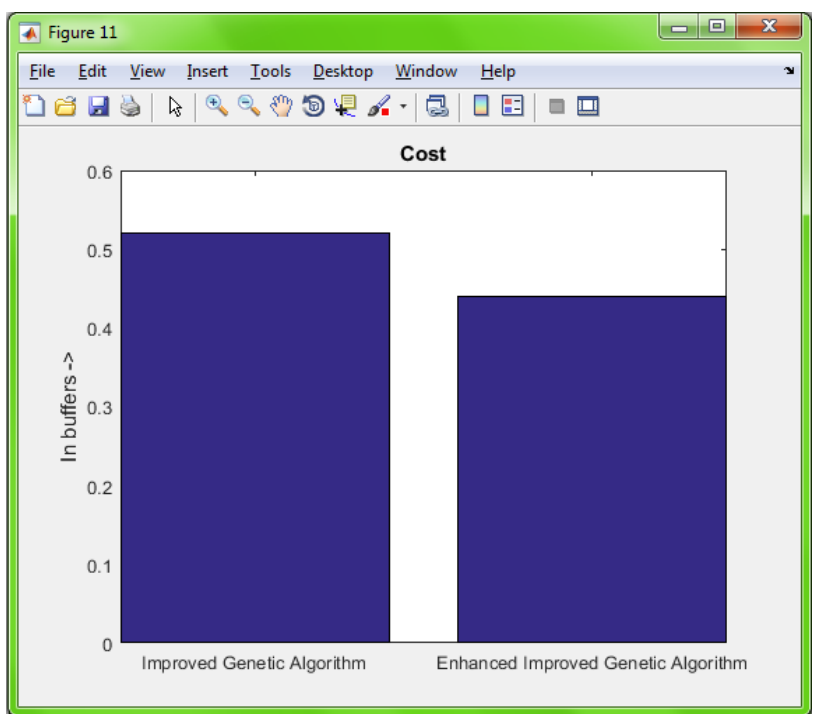

Fig 5: Comparison graph of Cost

Figure 5 shows the cost of the improved genetic algorithm and enhanced proposed improved genetic algorithm compared for the performance analysis. The enhanced improved genetic algorithm has less cost as compared to improved genetic algorithm.

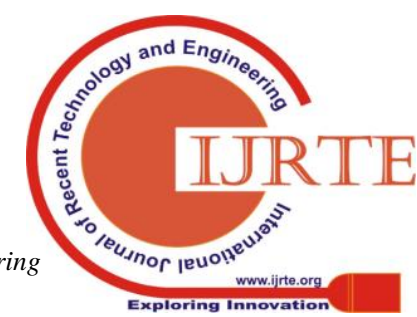




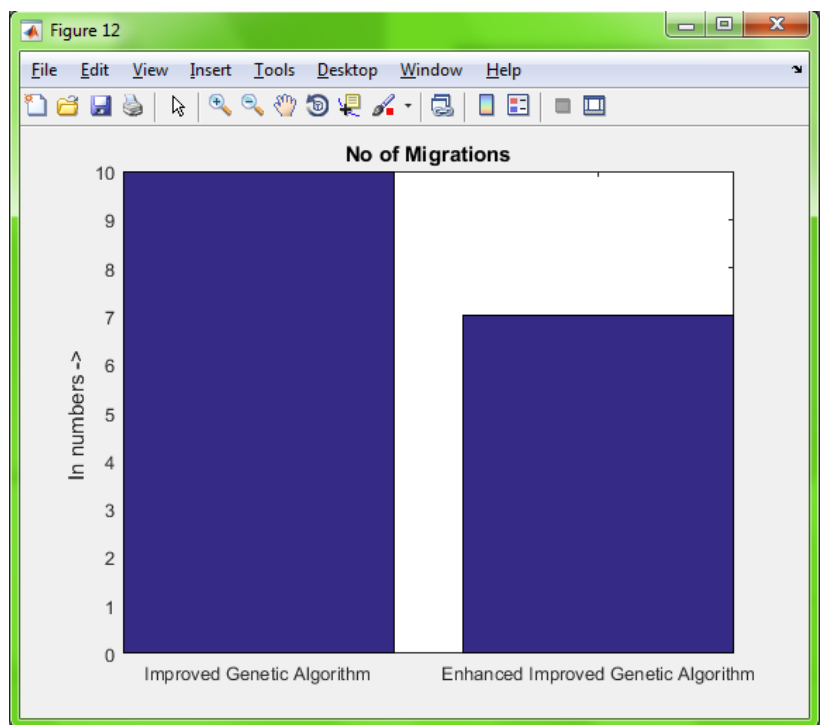

Fig 6: Comparison graph of No of Migrations

Figure 6 shows the number of migrations of improved genetic and proposed enhanced improved genetic algorithm compared for the performance analysis. The number of migration of enhanced improved genetic algorithm is less as compared to improved genetic algorithm.

Limitations

Following are the various limitations of this research work:

1. The cloud computing environment is the dynamic network due to which load balancing is the general problem which affects network performance. In the previous study, various techniques are proposed to balance network load. The existing techniques for load balancing require hardware and software which increase system complexity. The technique is required which does not need any extra hardware and software to network load.

2. The techniques which are proposed in existing work require extra steps to balance network load. The technique needs to be proposed which does not require extra steps to balance network load.

\section{FutUre SCOPE}

In future, the proposed algorithm can be further merged and compared with other algorithms of virtual machine migration and optimization can be done by using hybrid approach of meta- heuristic algorithm.

\section{CONCLUSION}

The cloud computing has the dynamic nature and due to which cloud network has various issues like security, quality of service and fault occurrence etc. The load balancing is the major issue of cloud network which reduce its efficiency. The algorithm that is imposed on existing work in cases when faults are detected to perform virtual machine migration is known as enhanced genetic algorithm. This work proposed a modification in the improved genetic algorithm such that the execution time can be minimized. The reliability and speed of the proposed algorithm are high due to which the chances that the fault will occur are minimized. MATLAB simulator is used to implement the proposed and existing algorithms. Comparisons amongst these two algorithms are made to evaluate their performances in terms of response time, finish time, energy consumption, cost and no of migrations. It is concluded that the proposed enhanced improved genetic algorithm shows high performance as compared to existing improved genetic algorithm for virtual machine migration.

\section{REFERENCES}

[1] Srinivas.J, K. Venkata Subba Reddy, Dr. A. Moiz Qyser, "Cloud Computing Basics", International journal of advanced research in computer and communication engineering, 2012, pp. 343-347.

[2] Soumya Ray and Ajanta De Sarkar, "Execution Analysis of Load Balancing Algorithm in Cloud computing Environment", International Journal on Cloud Computing: Services and Architecture (IJCCSA), October 2012, Vol.2, No.5

[3] HU Baofang, SUN Xiuli, LI Ying, SUN Hongfeng, “An Improved Adaptive Genetic Algorithm in Cloud Computing", 13th International Conference on Parallel and Distributed Computing, Applications and Technologies, 2012.

[4] Tushar Desai, Jignesh Prajapati, "A Survey of Various Load Balancing Techniques and Challenges in Cloud Computing", International Journal of Scientific \& Technology Research, November 2013, Volume 2, Issue 11.

[5] Punithasurya K, Esther Daniel, Dr. N. A. Vasanthi, "A Novel Role Based Cross Domain Access Control Scheme for Cloud Storage", International Journal of Advanced Research in Computer Engineering \& Technology (IJARCET), 2013, Volume 2, Issue 3, March 2013, pp 942-946.

[6] Vimmi Pandey, "Securing the Cloud Environment Using OTP", International Journal of Scientific Research in Computer Science and Engineering, 2013, vol-1, Issue-4.

[7] Sanjoli Singla, Jasmeet Singh, "Cloud Data Security using Authentication and Encryption Technique", International Journal of Advanced Research in Computer Engineering \& Technology (IJARCET), July 2013 Volume 2, Issue 7, pp 2232-2235.

[8] Sheetal Karki, Anshika Goyal, "Performance Evaluation of Check Pointing and Threshold Algorithm for Load Balancing in Cloud Computing", International Journal of Computer Sciences and Engineering, Vol.-6, Issue-5, May 2018, pp 2347-2693.

[9] Sukhpreet Kaur, Dr. Jyotsna Sengupta, "Load Balancing using Improved Genetic Algorithm(IGA) in Cloud Computing", International Journal of Advanced Research in Computer Engineering \& Technology (IJARCET), Volume 6, Issue 8, August 2017, pp 2278-1323.

[10] Wang Bei, LI Jun, "Load Balancing Task Scheduling based on Multi-Population Genetic Algorithm in Cloud Computing", 2016, Proceedings of the 35th Chinese Control Conference

[11] Mahalingam, Nandhalakshmi Nithya, "Efficient Load Balancing in Cloud Computing Using Weighted Throttled Algorithm", International Journal of Innovative Research in Computer and Communication Engineering, 2015,vol.3, $5409-5415$.

[12] Keke Gai, Meikang Qiu, Hui Zhao, "Cost-Aware Multimedia Data Allocation for Heterogeneous Memory Using Genetic Algorithm in Cloud Computing", IEEE Transactions on Cloud Computing, 2015.

[13] Mr. Mayur S. Pilavare, Mr. Amish Desai, “A Novel Approach Towards Improving Performance of Load Balancing Using Genetic Algorithm in Cloud Computing", IEEE Sponsored 2nd International Conference on Innovations in Information Embedded and Communication Systems, ICIIECS'15, 2015. 